\title{
Bacteria and Phagocytes: Mortal Enemies
}

\author{
Victor Nizet \\ Department of Pediatrics, School of Medicine and Skaggs School of Pharmacy and Pharmaceutical Sciences, \\ University of California San Diego, La Jolla, Calif., USA
}

A critical first-line element of mammalian innate immunity is the function of phagocytic cells, in particular neutrophils and macrophages. The effectiveness of these specialized leukocytes in host defense reflects their capacity for directed migration, microbial uptake, and intra- and extracellular microbial killing; the latter is achieved through the concerted action of reactive oxygen species, enzymatic proteolysis, and cationic antimicrobial peptides. Stimulated phagocytes also amplify inflammatory and immune responses through the release of cytokines, nitric oxide, and vasoactive peptides. Their general importance is further exemplified by the increased susceptibility to invasive bacterial infection in patients whose phagocyte numbers are markedly reduced. However, it is also apparent that several leading bacterial pathogens such as Staphylococcus aureus, Streptococcus pyogenes, and others are capable of causing severe invasive infections even in previously healthy individuals. Such intrinsic disease-producing capacity defines a superior ability of these pathogens to resist host phagocytic clearance through the coordinated expression of virulence determinants that interfere with phagocyte trafficking or opsonophagocytosis, or instead to neutralize the molecular effectors of bacterial killing. This special thematic issue of the Journal of Innate Immunity focuses on the elucidation of new bacterial virulence factors that target phagocyte defense pathways, pointing out the significant consequences they hold for the pathogen-host encounter.

\section{KARGER \\ Fax +4161306 1234 E-Mail karger@karger.ch} www.karger.com

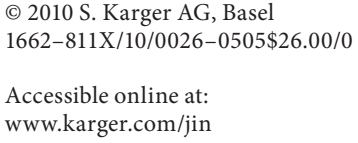

The research presented in this issue finds historical resonance in a classical scientific debate. Russian biologist Ilya Metchnikoff $[1,2]$ was awarded the Nobel Prize in 1908 for the development of the phagocyte theory, the central tenet of which was that the 'swallowing and digestion' of bacteria by circulating 'white corpuscles' (neutrophils and macrophages), enhanced by prior opsonization, provided immunity to infection [1-3]. However, a contemporary of Metchnikoff [1, 2], the hygienist and bacteriologist Oskar Bail [4], who was working at the German University (today named Charles University) in Prague, was simultaneously developing a countering 'aggressin doctrine'. This model cautioned that many pathogens, including $S$. aureus, pneumococcus, the tubercle bacillus, or cholera, were able to effectively neutralize phagocytes by paralysis of function or dissolution of the host cell, all through the action of specific 'aggressin' molecules induced and released during the course of infection. Metchnikoff [5] made a specific point in his Nobel Prize acceptance speech to trivialize Bail's [4] research: 'Numerous findings, achieved with care over the last few years, clean contradict this view. It has been shown that the white corpuscles entertain no fear of microbial poisons and are well fitted to absorb them and make them harmless.' As illustrated in this issue, the modern era of molecular microbiology has provided genetic approaches to pinpoint specific bacterial factors that fulfill the criteria of 'aggressin' proposed by Bail [4] - we can hope to the satisfaction rather than the chagrin of Metchnikoff's $[1,2]$ legacy.

Prof. Victor Nizet, Department of Pediatrics

School of Medicine, Skaggs School of Pharmacy and Pharmaceutical Sciences

University of California San Diego, 9500 Gilman Drive, Mail Code 0687

La Jolla, CA 92093-0687 (USA)

Tel. +1 858534 7408, Fax +1 858534 5611, E-Mail vnizet@ ucsd.edu 
After a phagocyte engulfs a bacteria, a critical ensuing step in the killing process is the fusion of the phagosome with cellular structures such as lysosomes (macrophages) or specific or azurophil granules (neutrophils) which can deliver cargos of hydrolytic enzymes, proteases, and cationic peptides with potent bactericidal properties. Here Cederlund et al. [6] provide a sophisticated analysis of the activities of several such bactericidal effectors, including lysosomes, $\alpha$-defensins, azurocidin, cathelicidin LL-37, calprotectin, and lactotransferrin, showing that specificity in the killing of different bacterial pathogens is provided by distinct repertoires of these bioactive peptides. One way for a bacterial pathogen to resist phagocyte killing is thus to prevent phagosome fusion with lysosomes and granules. Huynh et al. [7] demonstrate that the opportunistic pathogen Burkholderia cepacia is able to impair the activation of Rab7, a small GTPase that plays a key role in lysosome biogenesis and fusion with late endocytic structures [8]. For the invasive M1 serotype strain of the pathogen S. pyogenes, Hertzén et al. [9] show that the surface-expressed M1 protein prevents the fusion of the phagosome with the lysosome and provides the bacteria with an intracellular safe haven, creating a reservoir of surviving bacteria that can subsequently egress the macrophage to reinfect new cells.

S. aureus, a leading cause of serious bacterial infections and increasing antibiotic resistance, is capable of promoting the rapid elimination of human neutrophils. One key mechanism appears to be the action of staphopain $\mathrm{B}$, a cysteine protease that selectively cleaves CD11b on phagocytic cells; this is a 'cell death signal' which promotes rapid clearance of the affected neutrophils by macrophages thus reducing the number of phagocytes at the site of infection $[10,11]$. In this issue, 2 articles explore rapid neutrophil lysis induced by the epidemic USA300 clone of methicillin-resistant $S$. aureus (MRSA). Pang et al. [12] report that a global regulator of virulence factors, the agr system, is activated upon phagocytosis of USA300 MRSA leading to the upregulated expression of the poreforming $\alpha$-hemolysin within the phagosome and contributing to neutrophil destruction in as little as $2 \mathrm{~h}$. Kobayashi et al. [13] use live-cell imaging, electron microcopy, and whole-genome expression analysis to reveal that both low- and high-hemolysin-producing strains can induce such rapid neutrophil death. In these studies, neutrophil phagosome membranes appear to remain intact right up to the point of cell lysis, suggesting that USA300 MRSA triggers a novel form of programmed cell necrosis, a signature phenotype for its hypervirulence.
An important emerging concept in the field of neutrophil biology is that phagocytosis is not the sine qua non of bacterial killing. Rather, neutrophils can themselves undergo a specialized form of cell death that enhances innate immunity - the formation of neutrophil extracellular traps or NETs [14]. These structures comprise a backbone lattice of nuclear or mitochondrial DNA decorated with antimicrobial peptides, histones, and granule proteases that can ensnare and kill bacterial pathogens $[15,16]$. Berends et al. [17] report that $S$. aureus can thwart this extracellular killing mechanism by the elaboration of secreted nucleases that degrade the DNA backbone to dissolve the NETs. S. pyogenes strongly stimulates NET production by the proinflammatory action of its M1 protein in complex with fibrinogen [18], yet it can resist entrapment and killing by nuclease production [19] or binding and inactivation of the embedded cathelicidin antimicrobial peptides mediated by the M1 protein [20]. In the present issue, Pence et al. [21] use targeted mutagenesis and heterologous gene expression to show that the streptococcal inhibitor of complement (SIC), expressed by M1 strains of S. pyogenes, also inhibits cathelicidin killing to promote phagocyte resistance and systemic virulence. SIC, nuclease, capsule, and other neutrophil resistance factors of M1 S. pyogenes are upregulated in vivo as the result of selective pressure for mutations in the $\operatorname{cov} R S 2$-component regulator. Maamary et al. [22] show that a reduced propensity for covRS mutation predicts the increased neutrophil susceptibility and lower invasive disease potential of non-M1 S. pyogenes strains.

More than a century ago, Bail [4] foresaw that the neutralization of bacterial 'aggressins' could represent an important aspect of infectious disease therapy. While some of the virulence mechanisms discussed herein (e.g. S. aureus $\alpha$-hemolysin or staphopain B) do lead to the aggressive destruction of the phagocytic cell, others (e.g. S. pyogenes M1 protein or SIC and S. aureus nuclease) simply allow the pathogen to withstand the antimicrobial killing mechanisms deployed by the phagocyte and continue its replication in vivo (Author's note: perhaps virulence factors in the latter category might lightheartedly be referred to as 'passive aggressins').

The life-or-death battles between bacterial pathogens and host phagocytes will remain a critical determinative factor dictating infectious disease resolution or progression. Identification of the molecular effectors of bacterial phagocyte resistance can thus provide the novel opportunities for therapy suggested by Bail [4]. In this context, the therapeutic agent is not a classical antibiotic designed 
to kill the microbe directly, but rather a drug that 'disarms' the pathogen facilitating clearance by the host's normal phagocytic cell defenses. Proof-of-principle examples include a drug that blocks synthesis of the golden staphyloxanthin pigment of $S$. aureus, thus increasing the sensitivity to phagocyte oxidative burst [23], a molecule that inhibits protein kinase $\mathrm{G}$ of Mycobacterium tuberculosis to promote normal phagolysosome fusion [24], and an inhibitor of $S$. pyogenes DNase that preserves NETs and extracellular bacterial killing [19]. Continued elucidation of pathways and virulence factors conferring phagocyte resistance, as illustrated by the studies of this issue, can serve as a template for a new generation of targeted antimicrobials with increased specificity and reduced selective pressure for the spread of antimicrobial resistance.

\section{References}

1 Metchnikoff I: Ueber den Kampf der Zellen gegen Erysipelkokken, ein Beitrag zur Phagocytenlehre. Arch Pathol Anat (Virchow's Archiv) 1887;107:209-249.

2 Metchnikoff I: Immunity in Infective Diseases. Cambridge, Cambridge University Press, 1905.

3 Tauber AI: Metchnikoff and the phagocytosis theory. Nat Rev Mol Cell Biol 2003;4:897901.

4 Bail O: Das Problem der Bakteriellen Infektion. Leipzig, Klinkhardt, 1911.

5 Nobelprize.org: The Nobel Prize in Physiology or Medicine 1908. http://www.nobelprize.org/nobel_prizes/medicine/laureates/1908/index.html.

6 Cederlund A, Agerberth B, Bergman P: Specificity in killing pathogens is mediated by distinct repertoires of human neutrophil peptides. J Innate Immun 2010;2:508-521.

7 Huynh KK, Plumb JD, Downey GP, Valvano MA, Grinstein S: Inactivation of macrophage Rab7 by Burkholderia cenocepacia. J Innate Immun 2010;2:522-533.

8 Bucci C, Thomsen P, Nicoziani P, McCarthy J, van Deurs B: Rab7: a key to lysosome biogenesis. Mol Biol Cell 2000;11:467-480.

9 Hertzén E, Johansson L, Wallin R, Schmidt H, Kroll M, Rehn AP, Kotb M, Mörgelin M, Norrby-Teglund A: M1-protein dependent intracellular trafficking promotes persistence and replication of Streptococcus pyogenes in macrophages. J Innate Immun 2010; 2:534-545.

10 Smagur J, Guzik K, Magiera L, Bzowska M, Gruca M, Thogersen IB, Enghild JJ, Potempa J: A new pathway of staphylococcal pathogenesis: Apoptosis-like death induced by staphopain B in human neutrophils and monocytes. J Innate Immun 2009;1:98-108.
11 Potempa J, Pike RN: Corruption of innate immunity by bacterial proteases. J Innate Immun 2009;1:70-87.

12 Pang YY, Schwartz J, Thoendel M, Ackermann LW, Horswill AR, Nauseef WM: agrdependent interactions of Staphylococcus aureus USA300 with human polymorphonuclear neutrophils. J Innate Immun 2010; 2:546-559.

13 Kobayashi SD, Braughton KR, PalazzoloBallance AM, Kennedy AD, Sampaio E, Kristosturyan E, Whitney AR, Sturdevant DE, Dorward DW, Holland SM, Kreiswirth BN, Musser JM, Deleo FR: Rapid neutrophil destruction following phagocytosis of Staphylococcus aureus. J Innate Immun 2010;2: 560-575.

14 Brinkmann V, Reichard U, Goosmann C, Fauler B, Uhlemann Y, Weiss DS, Weinrauch Y, Zychlinsky A: Neutrophil extracellular traps kill bacteria. Science 2004;303:15321535.

15 Medina E: Neutrophil extracellular traps: a strategic tactic to defeat pathogens with potential consequences for the host. J Innate Immun 2009;1:176-180.

16 von Kockritz-Blickwede M, Nizet V: Innate immunity turned inside-out: antimicrobial defense by phagocyte extracellular traps. J Mol Med 2009;87:775-783.

17 Berends ETM, Horswill AR, Haste NM, Monestier M, Nizet V, von Köckritz-Blickwede M: Nuclease expression by Staphylococcus aureus facilitates escape from neutrophil extracellular traps. J Innate Immun 2010;2:576-586.

18 Oehmcke S, Morgelin M, Herwald H: Activation of the human contact system on neutrophil extracellular traps. J Innate Immun 2009;1:225-230.
19 Buchanan JT, Simpson AJ, Aziz RK, Liu GY, Kristian SA, Kotb M, Feramisco J, Nizet V: DNase expression allows the pathogen group A Streptococcus to escape killing in neutrophil extracellular traps. Curr Biol 2006;16: 396-400.

20 Lauth X, von Kockritz-Blickwede M, McNamara CW, Myskowski S, Zinkernagel AS, Beall B, Ghosh P, Gallo RL, Nizet V: M1 protein allows group A streptococcal survival in phagocyte extracellular traps through cathelicidin inhibition. J Innate Immun 2009;1: 202-214.

21 Pence MA, Rooijakkers SHM, Cogen AL, Cole JN, Hollands A, Gallo RL, Nizet V: Streptococcal inhibitor of complement promotes innate immune resistance phenotypes of invasive M1T1 group A Streptococcus. J Innate Immun 2010;2:587-595.

22 Maamary PG, Sanderson-Smith ML, Aziz RK, Hollands A, Cole JN, McKay FC, McArthur JD, Kirk JK, Cork AJ, Keefe RJ, Kansal RG, Sun H, Taylor WL, Chhatwal GS, Ginsburg D, Nizet V, Kotb M, Walker MJ: Parameters governing invasive disease propensity of non-M1 serotype group A streptococci. J Innate Immun 2010;2:596-606.

23 Liu CI, Liu GY, Song Y, Yin F, Hensler ME, Jeng WY, Nizet V, Wang AH, Oldfield E: A cholesterol biosynthesis inhibitor blocks Staphylococcus aureus virulence. Science 2008;319:1391-1394.

24 Walburger A, Koul A, Ferrari G, Nguyen L, Prescianotto-Baschong C, Huygen K, Klebl B, Thompson C, Bacher G, Pieters J: Protein kinase $\mathrm{G}$ from pathogenic mycobacteria promotes survival within macrophages. Science 2004;304:1800-1804 